\title{
Intracranial hemorrhage and extensive cerebral venous thrombosis associated with ulcerative colitis
}

\author{
Muhammad Adrish MD ${ }^{1,2}$, Ryan Rios MD
}

\section{CASE PRESENTATION}

A middle-age man with history of ulcerative colitis (UC) maintained on certolizumab and 6-mercaptopurine in addition to prednisone for a recent flare presented with altered mental status and severe headache of $6 \mathrm{~h}$ duration. According to his family, he had been experiencing mild intermittent headaches for the previous four weeks without any fevers, chills, blurred vision or neck stiffness. He had no history of trauma or ill contacts. Neurological examination revealed an attentive male with fluent but nonsensical speech. Motor and sensory examinations were nonfocal. A computed tomography scan of the brain showed acute hemorrhage in left temporal lobe with surrounding vasogenic edema and mass effect on the underlying brain parenchyma and left lateral ventricle. A magnetic resonance venogram revealed absence of signal within the left vein of Labbe, left transverse and sigmoid sinus, and left internal jugular vein consistent with extensive cerebral venous thrombosis (CVT) (Figures 1 and 2). Anticoagulation was initiated. His mental status subsequently deteriorated and repeat brain computed tomography scan showed worsening hemorrhage with midline shift and

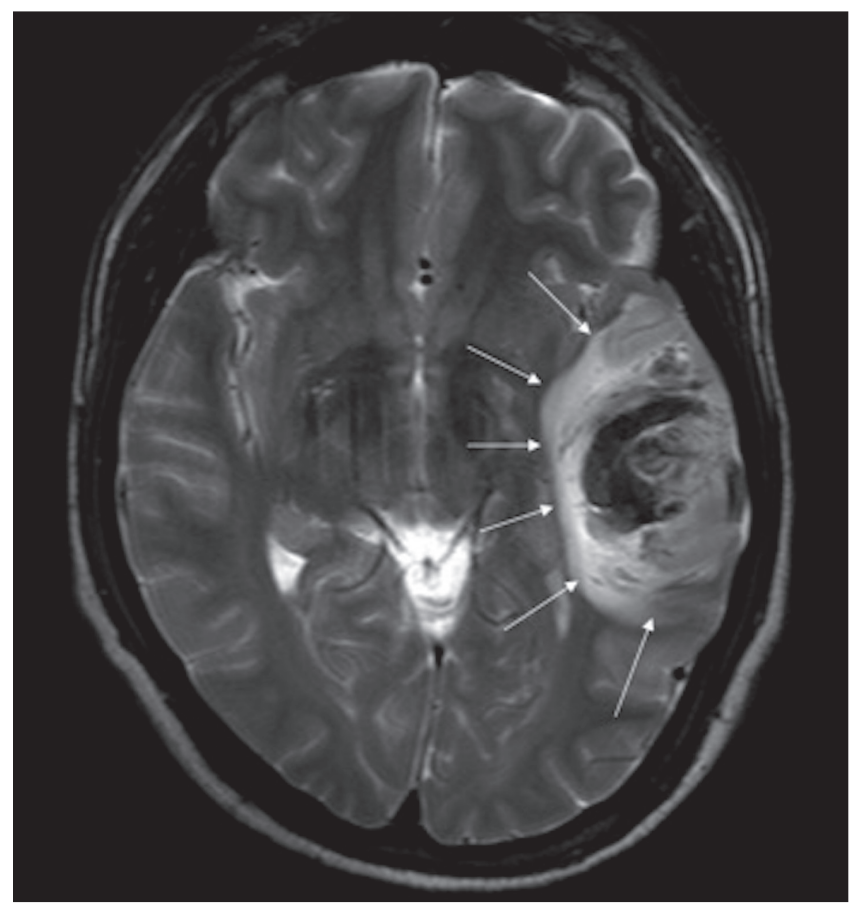

Figure 1) Magnetic resonance imaging. Axial T2 image showing heterogenous collection at the left temporal lobe consistent with a large intraparenchymal hemmorhage containing both subacute and acute blood. Moderate surrounding vasogenic edema (arrows) uncal herniation. The patient underwent emergent decompressive left hemicraniectomy. Anticoagulation was restarted postoperatively. The patient experienced a highly favourable recovery and was able to follow simple commands and move all extremities against gravity before his discharge from the hospital.

\section{DISCUSSION}

CVT is an uncommon disorder, with an annual incidence of three to four cases per million. Risk factors for CVT include thrombophilias, malignancies, hematological disorders, sinus infections, vasculatides, traumatic head injuries and inflammatory bowel diseases. Thrombosis of cerebral veins result in increased pressure in the venous system, which ultimately results in decreased cerebral perfusion, ischemic injury, disruption of the blood-brain barrier and vasogenic edema. Occasionally, this increased pressure can lead to venous and capillary rupture causing parenchymal hemorrhage. Clinical presentation of this entity is highly variable and can include headaches, seizures, encephalopathy or focal neurological signs. Diagnosis can be delayed for the same reason and, therefore, requires high clinical suspicion. Magnetic resonance venography is the most sensitive test for detection of CVT. Systemic anticoagulation is considered to be the primary

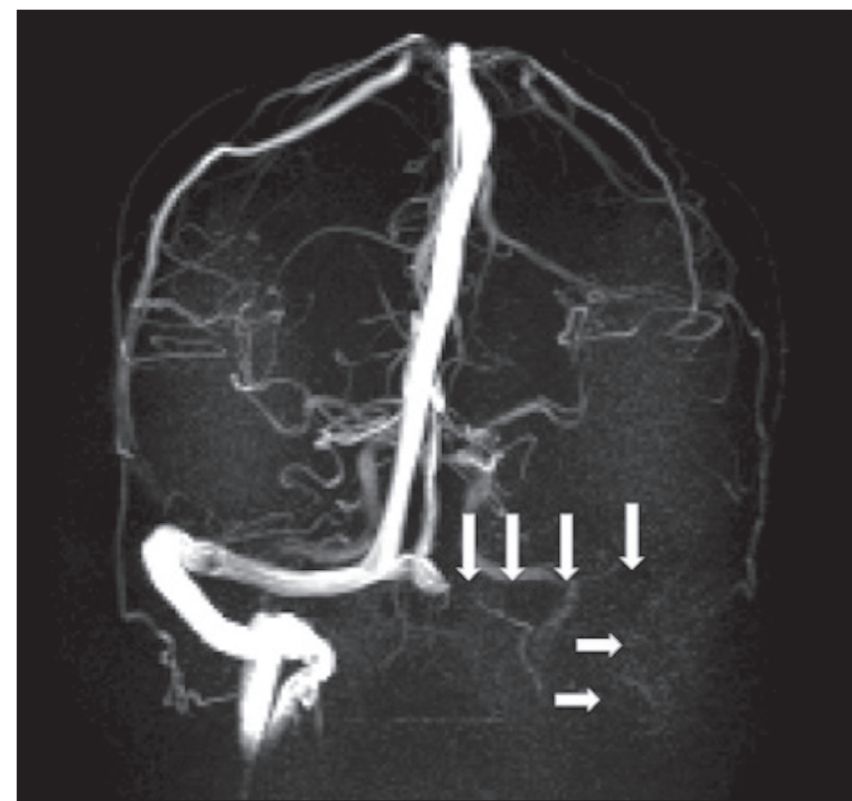

Figure 2) Magnetic resonance venogram image showing abrupt loss of flow related signal at the proximal left transverse sinus continuing distally to involve the left sigmoid sinus, vein of Labe and jugular vein (arrows)

${ }^{1}$ Department of Critical Care; ${ }^{2}$ Department of Medicine; ${ }^{3}$ Department of Neuroradiology, Montefiore Medical Center, Albert Einstein College of Medicine, Bronx, New York, USA

Correspondence: Dr Muhammad Adrish, Division of Critical Care Medicine, Montefiore Medical Center, Albert Einstein College of Medicine, 111 East $210 t h$

Street, Critical Care Administration, Bronx, New York 10467, USA. Telephone 917-375-2828, e-mail aadrish@hotmail.com

Received for publication March 31, 2014. Accepted April 28, 2014 
therapy for CVT even in individuals presenting with hemorrhagic infarcts $(1,2)$. In patients who do not respond to systemic anticoagulation or those presenting with severe neurological symptoms, catheterdirected thrombolytic therapy with or without thrombectomy may be considered (3). Herniation due to unilateral mass effect is a major cause of mortality and requires decompressive surgery. The majority of patients with CVT experience either complete or partial recovery, and recurrence is rare. In a meta-analysis of 1180 patients with CVT (4), the mean 30 -day mortality was $5.6 \%$.

DISCLOSURES: The authors report no conflict of interest.

\section{REFERENCES}

1. de Bruijn SF, Stam J. Randomized, placebo-controlled trial of anticoagulant treatment with low-molecular-weight heparin for cerebral sinus thrombosis. Stroke 1999;30:484-8.

2. Einhaupl KM, Villringer A, Meister W, et al. Heparin treatment in sinus venous thrombosis. Lancet 1991;338:597-600.

3. Canhao P, Falcao F, Ferro JM. Thrombolytics for cerebral sinus thrombosis: A systematic review. Cerebrovasc Dis 2003;15:159-66.

4. Dentali F, Gianni M, Crowther MA, Ageno W. Natural history of cerebral vein thrombosis: A systematic review. Blood 2006;108:1129-34.

The Canadian Journal of Gastroenterology $\mathcal{E}$ Hepatology is now considering a limited number of submissions for IMAGE OF THE MONTH. These are based on endoscopic, histological, radiological and/or patient images, which must be anonymous with no identifying features visible. The patient must consent to publication and the consent must be submitted with the manuscript. All manuscripts should be practical and relevant to clinical practice, and not simply a case report of an esoteric condition. The text should be brief, structured as CASE PRESENTATION and DISCUSSION, and not more than 700 words in length. A maximum of three images can be submitted and the number of references should not exceed five. The submission may be edited by our editorial team. 


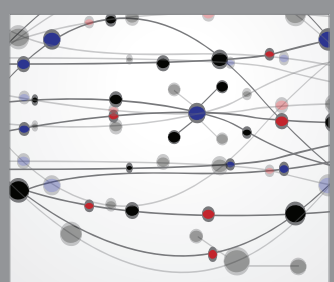

The Scientific World Journal
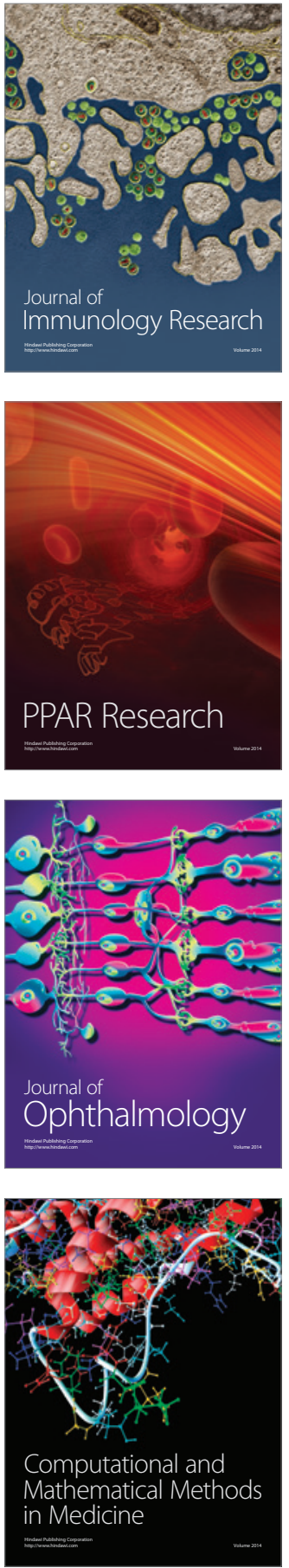

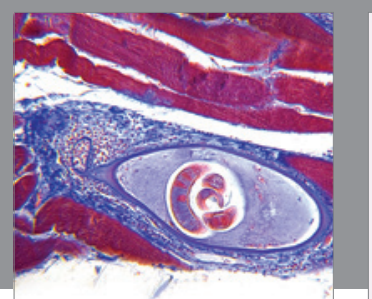

Gastroenterology Research and Practice

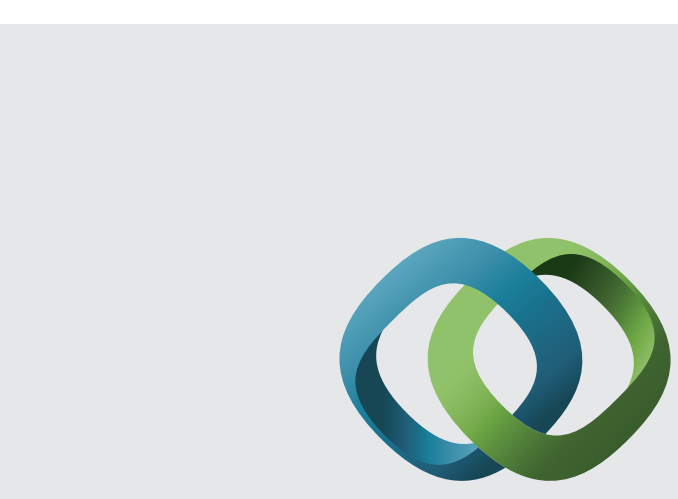

\section{Hindawi}

Submit your manuscripts at

http://www.hindawi.com
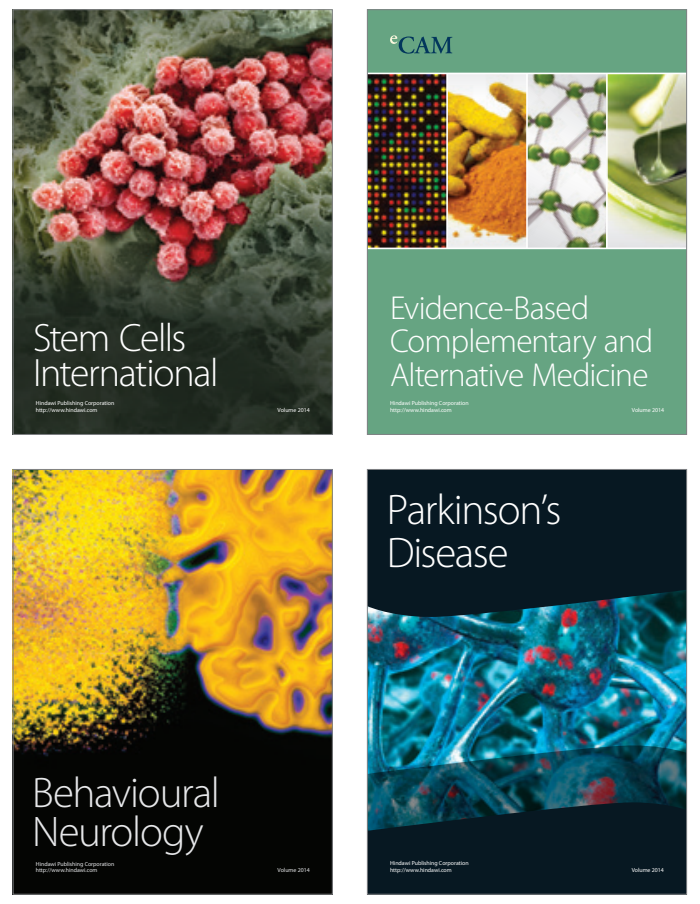
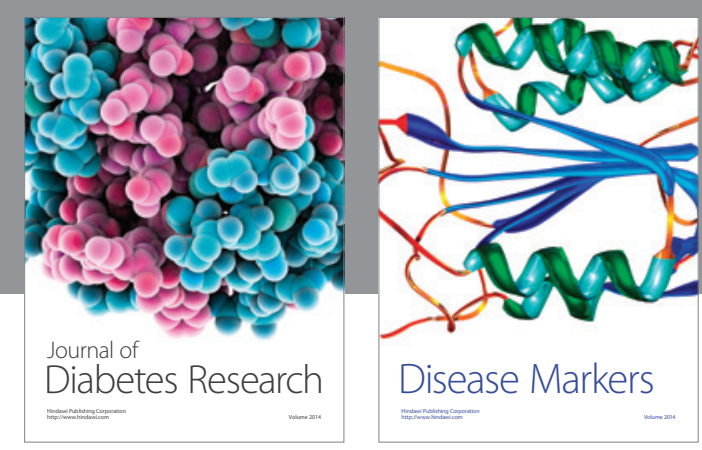

Disease Markers
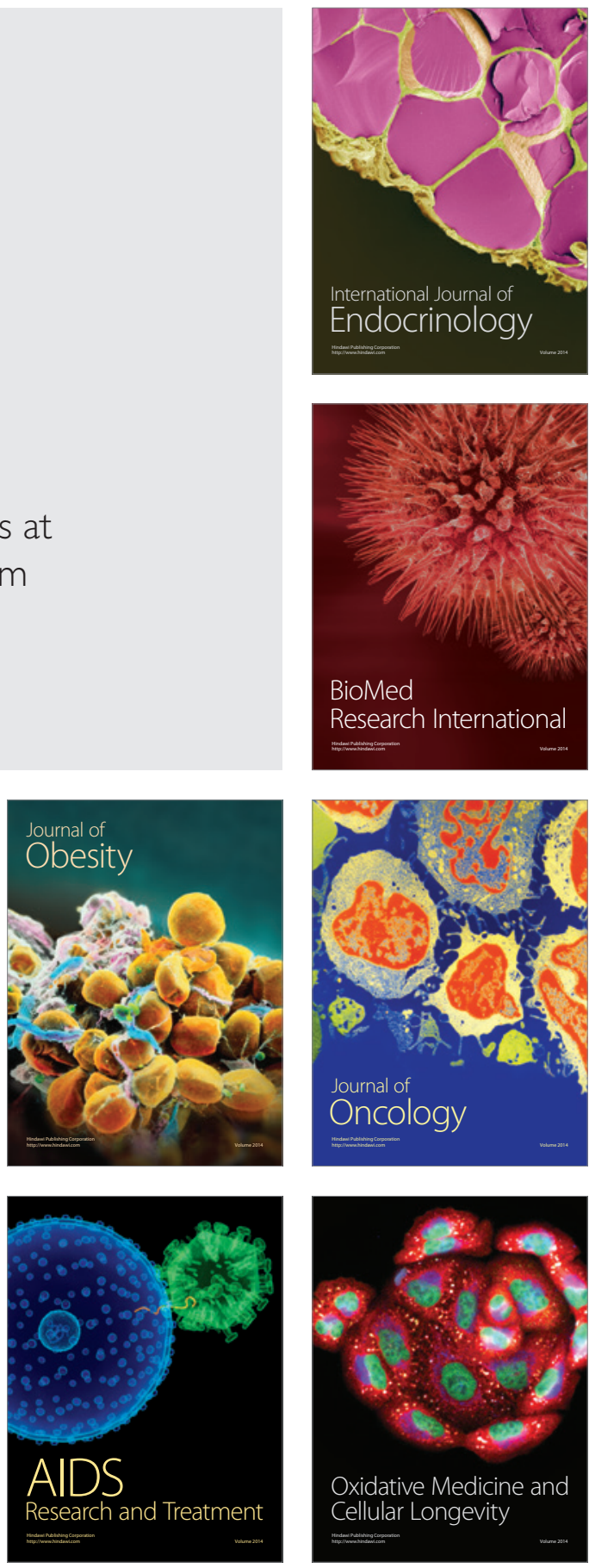